\title{
Intramural left coronary artery associated with right ventricular outflow tract obstruction
}

\author{
Sanjiv K. Gandhi, MD, Frank A. Pigula, MD, and Ralph D. Siewers, MD, Pittsburgh, Pa
}

A nomalies of the coronary arteries are well-described associations with tetralogy of Fallot. ${ }^{1-3}$ However, the presence of an intramural left coronary artery in combination with pathology involving right ventricular outflow tract obstruction has not been described. We describe 2 children with the anatomic complex of right ventricular outflow tract obstruction and an intramural, juxtacommissural, stenotic left coronary artery. The diagnosis was unknown in both children at the time of surgical intervention, and mortality resulted in both as a consequence of the anomaly.

\section{Clinical Summaries}

PATIENT 1. A 3.1-kg female infant born at term was transferred to our institution with cyanosis, which was stabilized with the infusion of epoprostenol (prostaglandin $\mathrm{E}_{1}$ ). Echocardiography confirmed the presence of severe pulmonary stenosis. Mild aortic stenosis and mitral stenosis were also present. The child had phenotypic characteristics suggestive of Noonan syndrome. A balloon pulmonary valvotomy was performed; however, there was only modest improvement in the gradient across the outflow tract. The pulmonary valve was noted to be extremely thick and doming, and the pulmonary annulus was only $4 \mathrm{~mm}$ in diameter. Although the baby remained stable after valvotomy, the systemic saturations remained in the low 60s. For this reason, the baby underwent a pulmonary valvectomy and patch enlargement of the right ventricular outflow tract with autologous pericardium at 2 weeks of age. The procedure was performed uneventfully during cardiopulmonary bypass without crossclamping the aorta. Weaning from bypass was met with severe systemic hypotension. Intraoperative echocardiography demonstrated severe global left ventricular (LV) dysfunction. Examination of the epicardial coronary arteries revealed poor filling of the entire left system. After surgical impingement of the left coronary system was excluded, the heart was arrested, and the coronary ostia were examined through an aortotomy. A very stenotic (0.5-mm orifice diameter), juxtacommissural, intramural left coronary artery was discovered. Unroofing of

From the Division of Pediatric Cardiothoracic Surgery, Department of Surgery, Children's Hospital of Pittsburgh, University of Pittsburgh School of Medicine, Pittsburgh, Pa.

Received for publication Aug 23, 2002; accepted for publication Sept 9, 2002.

Address for reprints: Sanjiv K. Gandhi, MD, Division of Cardiothoracic Surgery, Children's Hospital of Pittsburgh, 3705 Fifth Ave, Pittsburgh, PA 15213 (E-mail: sanjiv.gandhi@chp.edu).

J Thorac Cardiovasc Surg 2003;125:729-30

Copyright (C) 2003 by The American Association for Thoracic Surgery $0022-5223 / 2003 \$ 30.00+0$

doi: $10.1067 / \mathrm{mtc} .2003 .51$ this artery was performed from within the aorta. After repair, a $1.5-\mathrm{mm}$ probe was easily passed into the vessel. The patient could subsequently be weaned from bypass but required significant inotropic support to maintain an adequate systemic blood pressure. There was echocardiographic evidence of some improvement in LV function; however, the contractility was still significantly decreased. The decision was made to temporarily support the circulation with extracorporeal membrane oxygenation (ECMO) until ventricular contractility improved. Over the next 7 days, LV function recovered, and the patient was decannulated. However, approximately 8 hours after decannulation, despite adequate LV function, ECMO was reinstituted because of progressive hypotension and systemic acidosis. Surveillance blood cultures from the day before and the day of decannulation ultimately revealed staphylococcal sepsis. The patient subsequently had multiorgan dysfunction, and support was withdrawn on postoperative day 14 . Postmortem examination revealed a widely patent right ventricular outflow tract and confirmed the previously diagnosed additional valvular abnormalities. The right ventricle was small but not diminutive. The unroofed left main coronary artery was patent.

PATIENT 2. A 1-month-old, 3.5-kg male infant diagnosed with tetralogy of Fallot underwent an uneventful left modified BlalockTaussig (BT) shunt after hypercyanotic spells. He was discharged from the hospital on postoperative day 3 . Five weeks postoperatively, he experienced feeding difficulties and was admitted to the hospital. Within hours, he experienced hemodynamic collapse and required mechanical ventilation and high-dose inotropic support. Echocardiography demonstrated an occluded BT shunt and severe LV dysfunction. Subsequent cardiac catheterization was performed. Patency of the BT shunt was re-established with balloon angioplasty techniques, and no anatomic irregularities of the shunt were noted (Figure 1, A). However, the LV function was extremely decreased, and a normal left coronary artery was not visualized. A threadlike left coronary artery was seen to arise from the aorta (Figure 1, B). No coronary artery was seen to emanate from the pulmonary artery. The patient continued to decompensate hemodynamically and was placed on ECMO support. Cardiac transplantation was declined as an alternative by the family. The patient was successfully weaned from ECMO support and extubated, but the LV function never significantly recovered, the left ventricle continued to dilate, and the baby experienced refractory ventricular arrhythmias. The family ultimately discontinued support 4 weeks after ECMO decannulation. Postmortem examination revealed a tiny left coronary orifice located in a juxtacommissural position and taking an intramural course (Figure 2), explaining both the angiographic picture and the patient's clinical course.

\section{Discussion}

The anatomic association of right ventricular outflow tract obstruction and an intramural left coronary artery has not previously been described. We detail 2 children with this inordinately rare ana- 

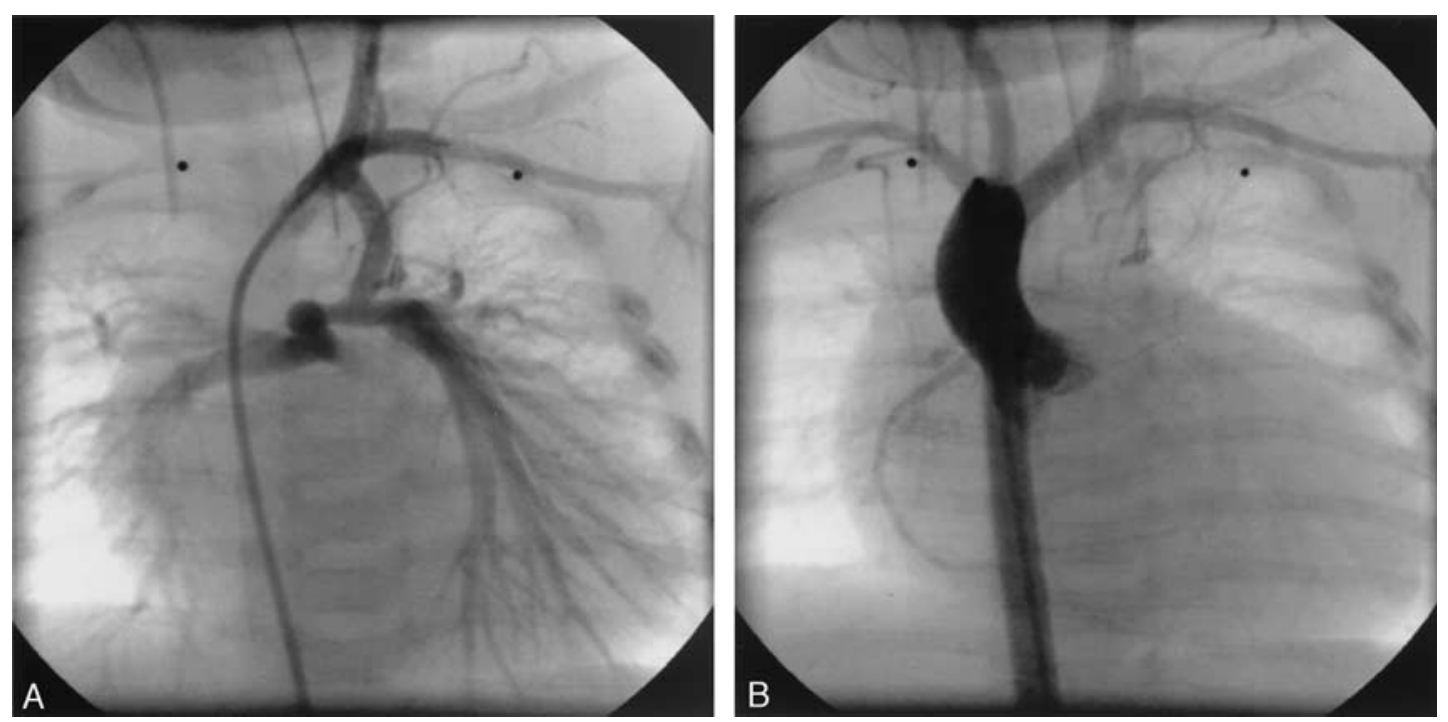

Figure 1. A, Angiogram demonstrating a patent BT shunt. B, Angiogram demonstrating poor filling of the left coronary artery.

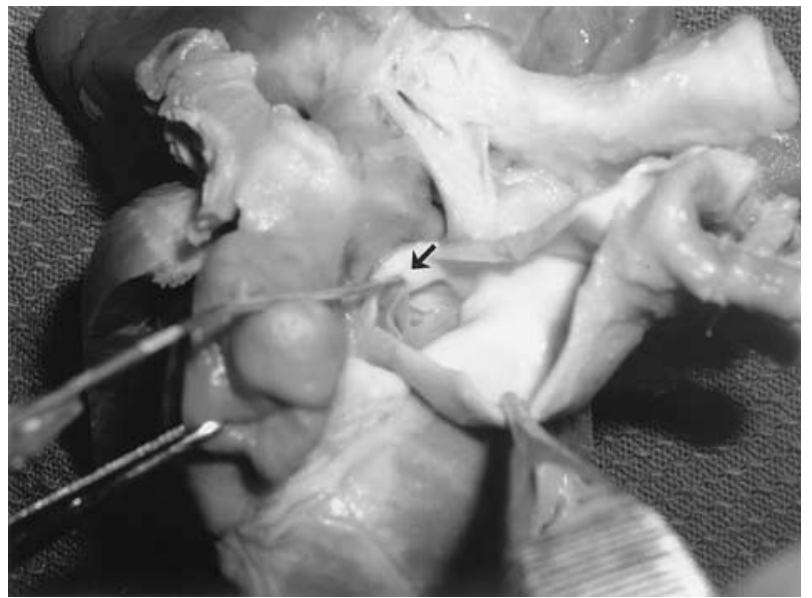

Figure 2. Postmortem specimen showing a stenotic, juxtacommissural, intramural coronary artery. A 1-mm probe (arrow) is seen to enter the stenotic orifice.

tomic association. The failure to diagnose the entity preoperatively is somewhat explained by its rarity but also by the difficulty in securing the diagnosis by means of either echocardiography or cardiac catheterization. In patient 1 there was no clinical suspicion preoperatively that there was a coronary problem; only after the stress of cardiopulmonary bypass did the problem become evident. Despite its recognition intraoperatively and expeditious surgical correction, persistent LV dysfunction mandated a period of extracorporeal support. Despite marked recovery of ventricular function, sepsis coupled with concomitant cardiac lesions, including a small right ventricle, ultimately led to the baby's death.

In patient 2 an echocardiogram and catheterization after the baby presented with hemodynamic collapse were unable to defin- itively demonstrate the pathology. Even had it been recognized, an attempt at surgical correction might have been futile given the clinical state of the patient. However, had the condition been known initially, it almost certainly would have altered the initial surgical management (ie, total correction versus performance of an interim palliative shunt procedure).

Anomalous origin of the left coronary artery with an intramural course has been described with a variety of congenital heart lesions. ${ }^{4,5}$ Successful repair techniques have also been performed. ${ }^{6}$ As with these disease entities, an effort to visualize the coronary origins in cases of right ventricular outflow tract obstruction should be made preoperatively. Cardiac catheterization with aortic root injection should be considered before surgical intervention in the neonatal period.

\section{References}

1. Davis JT, Teske DW, Allen HD, Cohen DM, Schauer GM. Anomalous course of the left main coronary artery in tetralogy of Fallot. Ann Thorac Surg. 1996;61:229-31.

2. Dabizzi RP, Teodori G, Barletta GA, Caprioli G, Baldrighi G, Baldrighi V. Associated coronary and cardiac anomalies in the tetralogy of Fallot. An angiographic study. Eur Heart J. 1990;11:692-704.

3. Mahant TS, Bajaj R, Shrivastava S, Goel PK. Anomalous left circumflex in tetralogy of Fallot—a case report. Int J Cardiol. 1995;48:18791.

4. Asou T, Karl TR, Pawade A, Mee RB. Arterial switch: translocation of the intramural coronary artery. Ann Thorac Surg. 1994;57:461-5.

5. Barbero-Marcial M, Tanamati C, Atik E, Ebaid M, Jatene A. Anomalous origin of the left coronary from the pulmonary artery with intramural aortic route: diagnosis and surgical treatment. $J$ Thorac Cardiovasc Surg. 1999;117:823-5.

6. Nakajima H, Yagihara T, Uemura H, Kawahira Y, Yoshikawa Y. Extended unroofing procedure for creation of a new ostium for anomalous left coronary artery. Ann Thorac Surg. 2001;72:1768-9. 